Check for updates

Cite this: RSC Adv., 2017, 7, 40734

\title{
Photodynamic antimicrobial chemotherapy with cationic phthalocyanines against Escherichia coli planktonic and biofilm cultures
}

\author{
Min Li, $\uparrow^{a}$ Bingjie Mai, $\uparrow^{a}$ Ao Wang, ${ }^{c}$ Yiru Gao, ${ }^{a}$ Xiaobing Wang, ${ }^{a}$ Xin Liu, ${ }^{a}$ \\ Shanshan Song, ${ }^{a}$ Quanghong Liu, ${ }^{a}$ Shaohua Wei $\mathbb{D}^{* b}$ and Pan Wang (D)*a
}

\begin{abstract}
Background and Objectives: Photodynamic antimicrobial chemotherapy (PACT) has emerged as a hopeful method for treating many bacteria-related infections. Phthalocyanines (Pcs) are promising photosensitizers with high photosensitivity. The aim of this study was to investigate the antimicrobial activity of new octacationic zinc phthalocyanines bearing 1,2-ethanediamine groups and the quaternized derivatives with different positive charges ( $\mathrm{ZnPc}^{n+}, n=4$ or 8 ) against Escherichia coli (E. coli) in both planktonic and biofilm states. Methods: The uptake of Pcs in E. coli was evaluated according to photometry after alkaline lysis. The dark-toxicity, light-toxicity and light-mediated antimicrobial effect of the drug were determined by plate count method. Intracellular reactive oxygen species (ROS) production was detected by flow cytometry. Scanning electron microscopy (SEM) and propidium iodide (PI) staining were performed to assess the disruption of the biofilm and membrane integrity, respectively. Results: With the incubation time prolonged, the relative fluorescence intensity of the two Pcs increased and peaked at 40 minutes. Pcs alone and irradiation itself had no evident toxicity to the bacteria while a remarkable survival decrease was observed in the PACT groups in a light dose-dependent manner. ZnPc1 showed a more than 3-log reduction while ZnPc2 caused a nearly 5-log reduction of bacterial counts. Intracellular ROS levels were significantly enhanced by PACT treatment. The disruption of the biofilm and membrane integrity detected by SEM and PI staining suggested that PCS-PACT can effectively damage the biofilm and cell membrane. Conclusion: All the results indicate that PCS-PACT presents excellent bactericidal activity.
\end{abstract}

Received 31st May 2017

Accepted 3rd August 2017

DOI: 10.1039/c7ra06073d

rsc.li/rsc-advances

\section{Introduction}

Previous research has shown that nearly one-third of global mortality arises from bacterial infections, which can cause a wide range of diseases including listeriosis, anthracnose, urinary tract infections, and gastroenteritis. ${ }^{1-3}$ Many therapies to cure bacterial infections have been explored over time. Conventional methods such as ultraviolet (UV) germicidal irradiation and thermotherapy could disinfect microorganisms, even from bacteria in biofilm-producing modes and viruses, but

${ }^{a}$ Key Laboratory of Medicinal Resources and Natural Pharmaceutical Chemistry, Ministry of Education, National Engineering Laboratory for Resource Developing of Endangered Chinese Crude Drugs in Northwest of China, College of Life Sciences, Shaanxi Normal University, Xi'an 710062, China. E-mail: wangpan@snnu.edu.cn; Tel: +86-29-8531-0275

${ }^{b}$ School of Chemistry and Materials Science, Jiangsu Key Laboratory of Biofunctional Materials, Jiangsu Collaborative Innovation Centre of Biomedical Functional Materials, Key Laboratory of Applied Photochemistry, Nanjing Normal University, Wenyuan Road No. 1, Nanjing 210023, China. E-mail: shwei@njnu.edu.cn 'Institute of Chemical Industry of Forest Products, Chinese Academy of Forestry, No. 16, Suojin 5th Village, Nanjing 210042, China

$\dagger$ The authors contributed equally to this work. have their drawbacks. For example, thermal and UV-based disinfections require excessive amounts of energy, and sometimes it is difficult to control experimental conditions to get a good result., ${ }^{4,5}$ Traditional antibiotics such as penicillin, oxacillin, and amoxicillin have specific intracellular targets; consequently, bacteria are able to develop resistance to these drugs. ${ }^{6}$ Especially, Gram-negative bacteria such as Escherichia coli $(E$. coli) are known to have an outer membrane in the cell wall structure that can serve as a protective barrier to control the influx and efflux of solutes, thus making it more difficult to disinfect the Gram-negative microbes with the same dosage of drug compared with Gram-positive ones. ${ }^{\mathbf{7 - 1 1}}$ Furthermore, many bacteria strains in the biofilm state are extremely recalcitrant to antimicrobials for it is more difficult to penetrate into the biofilm, which can produce extracellular polymeric substances (EPS) and accumulate to reach an effective concentration to kill them. ${ }^{12}$

A promising approach to treat bacterial infections is photodynamic antimicrobial chemotherapy (PACT), which was recognized at the start of the twentieth century and has been developed to treat both cancers and infections of pathogenic microorganisms, and has produced good results. ${ }^{13-16}$ This 
measure uses photosensitizers (PSs) to interact with light; this interaction results in the production of a kind of strong oxidant called reactive oxygen species (ROS), which are generated by energy or electron transfer from the PS short-lived excited single state to the PS long-lived excited triplet state. ${ }^{14}$ The targets of ROS are not only the cell membranes; they also destroy intracellular proteins and DNAs, so it is difficult for bacteria to develop resistance against PACT. ${ }^{\mathbf{1 3 , 1 4}}$

The PS is a crucial element in PACT, which can directly affect the efficiency. Porphyrins, chlorins, bacteriochlorins, methylene blue as well as a large number of dyes with different molecular frameworks have been proposed as antimicrobial PSs to be used in PACT. ${ }^{17,18}$ When we design or choose a photosensitizer for biomedical application, many factors, like singlet oxygen generation, water-solubility, and cellular uptake, should be taken into consideration. ${ }^{19}$ However, most used PSs are hydrophobic with poor solubility in water. As a result, they aggregate easily under physiological conditions, drastically lowering the quantum yields of ROS production and finally influencing the effect of PACT ${ }^{20,21}$ Furthermore, the presence of the outer membrane makes Gram-negative bacteria more resistant to photoinactivation than Gram-positive bacteria. ${ }^{22}$ Phthalocyanines (Pcs), which were first synthesized by chance in 1907, are remarkable macrocyclic compounds with magnificent physical and chemical properties, such as efficient singlet oxygen generation, high photo-toxicity, and high thermal and light stability. ${ }^{23}$ On account of these characteristics, Pcs have been regarded as the promising photosensitizers for PACT. ${ }^{23-25}$ However, it is a pity that Pcs cannot be widely used in PACT because of their low solubility and aggregation phenomena in water, which have a dramatic influence on their singlet oxygen $\left({ }^{1} \mathrm{O}_{2}\right)$ generation efficiency. ${ }^{23}$ Therefore, many researchers have tried to introduce hydrophilic groups, such as polyethylene glycol and ionic substituents, at the macrocycle periphery in order to decrease their aggregation and increase their solution in water. ${ }^{26}$ Lin et al. synthesized a kind of amino groupsubstituted cationic phthalocyanine derivative that was proved to have good solubility and anti-cancer activity, ${ }^{27}$ but its antibacterial effect was not evaluated. In addition, Durmuş et al. reported a type of quaternized mercaptopyridine-substituted zinc-phthalocyanine with excellent water solubility and anticancer activity, but its antimicrobial effect was not measured either. ${ }^{28}$ Other researchers working in related areas have reported a polyvalent methylsulfonyl zinc-phthalocyanine that possessed excellent phototoxicity towards Gram-positive Staphylococcus aureus ( $S$. aureus) but had no effect on Gramnegative $E$. coli even with a concentration at $100 \mu \mathrm{M} .{ }^{19}$ In this study, we used two octa-cationic zinc phthalocyanines bearing a 1,2-ethanediamine group and the quaternized derivatives, quaternized 2(3), 9(10), 16(17), 23(24)-tetra-(((2-dimethylamino) methyl)phenoxy)phthalocyaninato-zinc(II) (ZnPc1 or $\mathrm{ZnPc}^{4+}$ ) and quaternized 2(3), 9(10), 16(17), 23(24)-tetra-(((2-aminoethylamino)methyl)phenoxy)phthalocyaninato-zinc(II) (ZnPc2 or $\mathrm{ZnPc}^{8+}$ ), which have been proved to have good solubility, low aggregation, a high rate of singlet oxygen generation and high efficiency of intracellular uptake in our previous research. ${ }^{23}$ The purpose of our study was to investigate the antimicrobial activity of the two Pcs against $E$. coli after combining with light and make a brief comparison of them so as to lay a foundation for their clinical application in the future.

\section{Materials and methods}

\subsection{Bacterial strain and culture conditions}

Escherichia coli ( $E$. coli) was stored at $-80{ }^{\circ} \mathrm{C}$ as glycerol stocks. Before experiments, the strain was first activated in $37{ }^{\circ} \mathrm{C}$ water followed by inoculating on Trypticase Soy Agar (TSA, Aobox biotechnology, Beijing, China) for 24 hours. A colony was transferred into tryptic soy broth (TSB, Aobox biotechnology, Beijing, China) and grown overnight at $37{ }^{\circ} \mathrm{C}$ with shaking at a rate of $120 \mathrm{rpm}$ in an orbital shaker (TS-200B, TENSUC, Shanghai, China). Then the culture was centrifuged for 5 minutes at $9000 \mathrm{rpm}$ and diluted using PBS (phosphatebuffered saline) to a concentration of $2 \times 10^{8} \mathrm{CFU}$ (colonyforming units) per $\mathrm{ml}$ in PBS by determining the $\mathrm{OD}_{600}$ with a UV-visible spectrophotometer (SpectraMax M5, Molecular Devices, Sunnyvale, USA).

\subsection{Photosensitizers and light source}

The detailed synthesis of the two Pcs used in this study has been published before. ${ }^{23,27}$ A brief overview is shown below.

To synthesize ZnPc1, 4-(4-((dimethylamino)methyl)phenoxy) phthalonitrile (compound $\mathrm{A}_{2}$ ) was obtained originated from 4(4-(aminomethyl)phenoxy) phthalonitrile (compound $A_{1}$ ). After that, compound $\mathrm{A}_{2}$, zinc acetate, $n$-amyl alcohol and 1,8diazabicyclo[5,4,0]-undec-7-ene (DBU) were used to get 2(3), 9(10), 16(17), 23(24)-tetra-(((dimethylamino)methyl)phenoxy) phthalocyaninato-zinc(II) (ZnPcA). Next, $\mathrm{ZnPcA}$ and $\mathrm{CH}_{3} \mathrm{I}$ were dissolved in acetonitrile at reflux to obtain the crude product. The final product quaternized 2(3), 9(10), 16(17), 23(24)-tetra(((dimethylamino)methyl)phenoxy)phthalocyaninato-zinc(II) (ZnPc1) was collected by filtration and washed by diethyl ether, ethyl alcohol and acetone successively. ${ }^{27}$

As for the synthesis of $\mathrm{ZnPc2}$, 4-((2-(tritylamino)ethylamino) methyl)phenol (compound $\mathrm{B}_{2}$ ) was first synthesized from $\mathrm{N}$ tritylethane-1,2-diamine (compound $\mathrm{B}_{1}$ ). After that, $\mathrm{B}_{2}$ was used to obtain 4-(4-((2-(tritylamino)ethylamino)methyl)phenoxy) phthalonitrile (compound $\mathrm{B}_{3}$ ) by reacting with 4-nitrophthalonitrile. Next, we used compound $\mathrm{B}_{3}$, anhydrous zinc acetate and DBU to synthesize 2(3), 9(10), 16(17), 23(24)-tetra(((2-(tritylamino)ethylamino)methyl)phenoxy)phthalocyani-

nato-zinc(II) (ZnPcB). Finally, $\mathrm{ZnPcB}$ mixed with excess trifluoroacetic acid (TFA) in $\mathrm{CH}_{2} \mathrm{Cl}_{2}$ to get green solid product. The green solid was used to react with $\mathrm{K}_{2} \mathrm{CO}_{3}$ and $\mathrm{CH}_{3} \mathrm{I}$ under specific experimental conditions to get the final product quaternized 2(3), 9(10), 16(17), 23(24)-tetra-(((2-aminoethylamino) methyl)phenoxy)phthalocyaninato-zinc(II) (ZnPc2). ${ }^{23}$

The stock solutions of ZnPc1 and ZnPc2 dissolved in dimethyl sulfoxide (DMSO) with a concentration of $10 \mathrm{mM}$ and $5 \mathrm{mM}$, respectively, were stored at $4{ }^{\circ} \mathrm{C}$ in the dark. The structural formulae of the two Pcs are shown in Fig. 1.

The treatment of PACT in the following experiments was measured by a semiconductor laser (NingJu Photoelectric 

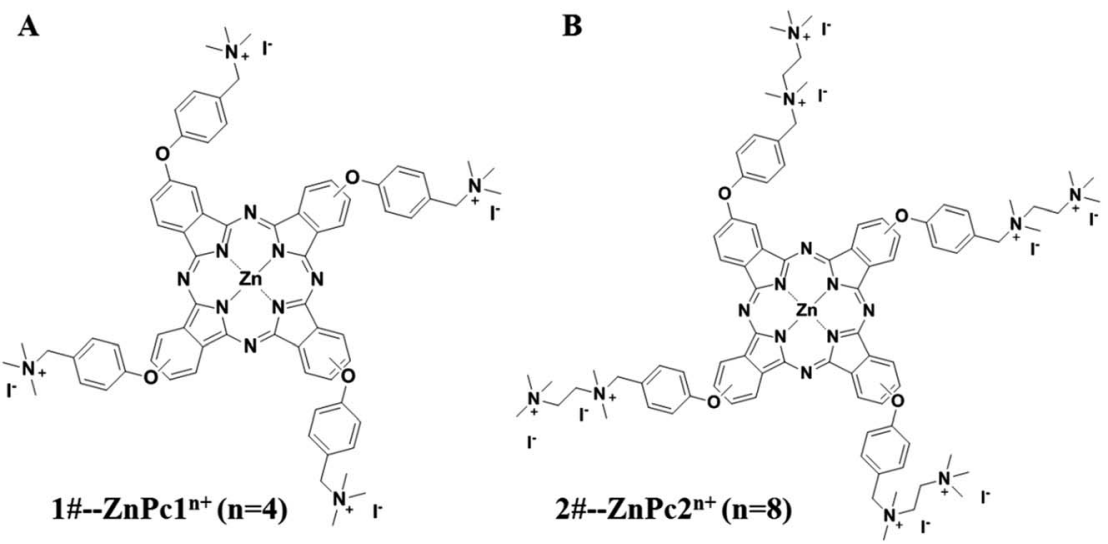

Fig. 1 The structural formulae of the phthalocyanines (Pcs). (A) 1\#-Pcs (ZnPc1) and (B) 2\#-Pcs (ZnPc2).

Technology Limited Company, Xi'an, China). Laser irradiance was measured using a radiometer system (NingJu photoelectric technology limited company, Xi'an, China). The laser was used with a power of $300 \mathrm{~mW}$ and an irradiation time of 0.1-5.8 minutes such that the final dose of light ranged from 1 to $50 \mathrm{~J}$ $\mathrm{cm}^{-2}$.

\subsection{The uptake of Pes}

The alkaline lysis method, a technique for the fast pyrolysis of bacteria, was used in this part. ${ }^{29}$ Firstly, the bacteria suspensions $\left(2 \times 10^{8} \mathrm{CFU} \mathrm{^{-1 } )}\right.$ were co-incubated with Pcs at a concentration of $5 \mu \mathrm{M}$ (selected from the pretest studies) in the dark at $37{ }^{\circ} \mathrm{C}$. At 20 minute intervals, the mixture was removed from the dark and a $1 \mathrm{ml}$ aliquot was taken out and centrifuged for 5 minutes at a speed of $9000 \mathrm{rpm}$. After this period, the pellet was washed twice with PBS and then the precipitate was lysed with $200 \mu$ l of buffer I (50 mM glucose, $25 \mathrm{mM}$ Tris-HCL, $10 \mathrm{mM}$ EDTA). 2 minutes later, the same volume of lysis buffer II ( $0.2 \mathrm{M} \mathrm{NaOH}, 1 \% \mathrm{SDS})$ was added. After 3 minutes standing, the samples were centrifuged at $9000 \mathrm{rpm}$ for 5 minutes. The fluorescence spectra of the supernatant were recorded between 650 and $710 \mathrm{~nm}$ by using a UV-visible spectrophotometer with the excitation wavelength at $345 \mathrm{~nm}$. The relative fluorescence intensity was represented as $F_{t}$ (after adding Pcs at time $t$ ) minus $F_{0}$ (before adding Pcs).

\subsection{Dark toxicity studies}

In order to investigate the dark toxicity of the two photosensitizers, the plate count method was used. ${ }^{30} E$. coli with a concentration of $2 \times 10^{8} \mathrm{CFU} \mathrm{ml}^{-1}$ was mixed with Pcs to give final concentration of the drugs of $0,5,10,20,50$, and $100 \mu \mathrm{M}$ for $\mathrm{ZnPc} 1$ and $0,2,5,10,20$, and $50 \mu \mathrm{M}$ for $\mathrm{ZnPc} 2$ (the different concentrations of two drugs were selected according to our preexperiments). Then the mixtures with different drug concentrations were added to 24 -well plates and placed in a dark humidified atmosphere of $95 \%$ air and $5 \% \mathrm{CO}_{2}$ at $37{ }^{\circ} \mathrm{C}$ to incubate for 40 minutes. After the desired incubation time, the culture was serially diluted ten-fold with PBS and then $100 \mu \mathrm{l}$ of the solution was coated on agar plates. Colonies that formed after 24 hours of incubation at $37{ }^{\circ} \mathrm{C}$ were counted.

\subsection{Light toxicity studies}

Considering whether the light irradiation (power of $300 \mathrm{~mW}$ ) alone would cause damage to the bacteria, the plate count method was carried out in this part. $E$. coli at a concentration of $2 \times 10^{8} \mathrm{CFU} \mathrm{ml}^{-1}$ was placed in 24 -well flat bottom plates and irradiated with light $\left(10,20,50,100,200 \mathrm{~J} \mathrm{~cm}^{-2}\right)$. The bacteria without irradiation were used as the control. After irradiation, the suspensions were serially diluted ten-fold with PBS and 100 $\mu \mathrm{l}$ aliquots were evenly coated on the agar plates for incubation in the incubator; after 24 hours the colonies were counted. In addition, cells treated with different doses of light irradiation were stained with propidium iodide (PI, Sigma, MO, USA) to detect the membrane integrity by fluorescence microscopy. ${ }^{31,32}$ Besides, we used infrared thermal imaging instrument (FLIR, FLIR-E6390, Estonia) to monitor the temperature change from 0 to 10 minutes during irradiation to determine whether the heating would disinfect the bacteria.

\subsection{Photodynamic antimicrobial activity of ZnPc1 and ZnPc2}

In this period, the plate count method was used, too. $E$. coli with a concentration of $2 \times 10^{8} \mathrm{CFU} \mathrm{ml}^{-1}$ was co-cultured with Pcs $(5,10$, and $20 \mu \mathrm{M}$ for ZnPc1 and 1, 2, and $5 \mu \mathrm{M}$ for ZnPc2) in the dark for 40 minutes in 24-well flat bottom plates. Subsequently, the plates were irradiated with laser light at different light doses $\left(10,30\right.$, and $50 \mathrm{~J} \mathrm{~cm}^{-2}$ for ZnPc1 and 10,20 , and $30 \mathrm{~J} \mathrm{~cm}^{-2}$ for ZnPc2). Aliquots without photosensitizers and irradiation were taken as the control. The suspensions in the wells were considered to be a dilution factor of $10^{-1} .1$ to 5 serial dilutions were then made using each original $10^{-1}$ dilution, and aliquots of $100 \mu \mathrm{l}$ were seeded onto agar plates followed by incubation at $37{ }^{\circ} \mathrm{C}$ for 24 hours. After the incubation period, the $\mathrm{CFU} \mathrm{ml} \mathrm{m}^{-1}$ values of each plate were determined.

\subsection{Flow cytometry to detect the intracellular ROS level}

Intracellular reactive oxygen species accumulation can be monitored by dihydroethidium (DHE, Vigorous, Beijing, 
China), which is sensitive to superoxide anions but is considered as a minor contributor to overall ROS. ${ }^{15}$ When it is absorbed into the cells, it can be dehydrogenized with the interaction of superoxide anions and produce ethidium, which can combine with DNAs or RNAs and cause the red fluorescence product 2-hydroxyethidium to accumulate in the cytoplasm rapidly. ${ }^{33,34}$ In order to detect the intracellular ROS level, the ROS probe DHE (stock solution of $5 \mathrm{mM}$ ) was added to bacterial suspensions with a concentration of $2 \times 10^{8} \mathrm{CFU} \mathrm{ml}^{-1}$. Then the mixture was incubated at $37{ }^{\circ} \mathrm{C}$ for 30 minutes followed by adding the sensitizers (ZnPc1 of $20 \mu \mathrm{M}$ and ZnPc2 of $5 \mu \mathrm{M}$ ) to the culture and then further incubated for another 40 minutes. After PACT treatment, bacteria were washed with PBS, and the fluorescent intensity in each group was immediately analyzed by flow cytometry (NovoCyte, ACEA Biosciences Inc, California, USA).

\subsection{Membrane integrity measurement}

To detect the membrane integrity after PACT treatment, PI staining was used in this part, as described previously. ${ }^{31,32}$ Bacteria suspensions with concentration of $2 \times 10^{8} \mathrm{CFU} \mathrm{ml}^{-1}$ were treated with Pcs-PACT and the plates were kept in the dark for 30 minutes. After that, PI (stock solution of $1 \mathrm{mg} \mathrm{ml}^{-1}$ ) was added to the culture with a volume ratio of $1: 100$ and then further incubated for another 15 minutes, and then the fluorescent signal of the cells in each group was quantified using flow cytometry. In addition, fluorescent images were taken using fluorescence microscope (Axio Imager M2, Zeiss, Oberkochen, Germany) to further prove the membrane damage.

\subsection{Scanning electron microscopy}

SEM was performed to analyze the effect of Pcs on the structural integrity of the bacterial biofilms. In this study, we used fresh TSB to adjust the bacteria to a liquid concentration of $1 \times 10^{7}$ CFU ml $\mathrm{ml}^{-1}$. Biofilms were grown on glass coverslips in 24-well plates for two days, and the medium was replaced every 24 hours. ${ }^{35}$ In order to enhance biofilm formation, the coverslips were precoated with $20 \%$ fetal bovine serum before adding the bacteria. Two days later, biofilms were treated with Pcs $(20 \mu \mathrm{M}$ for $\mathrm{ZnPc1}$ and $5 \mu \mathrm{M}$ for $\mathrm{ZnPc} 2)$ and then irradiated with different light doses $\left(10,30\right.$, and $50 \mathrm{~J} \mathrm{~cm}^{-2}$ for ZnPc1 and 10, 20, and $30 \mathrm{~J} \mathrm{~cm}^{-2}$ for ZnPc2). After treatment as described above, samples were washed several times and then fixed using 2.5\% glutaraldehyde in 0.1 M PBS (pH 7.2) for 4 hours at $4{ }^{\circ} \mathrm{C}$. Fixed samples were dehydrated in increasing concentrations of ethanol $(30 \%, 50 \%, 70 \%, 80 \%, 90 \%, 95 \%$ and $100 \%)$ for 10 minutes. After this period, the ethanol was displaced by isoamyl acetate. The coverslips were finally dried and mounted and then sputter coated with gold-palladium. Finally, the samples were analyzed under a scanning electron microscope (S-3400N, Hitachi, Tokyo, Japan).

\subsection{Statistical analysis}

SPSS 19.0 software (SPSS Inc., Chicago) was used for statistical analysis. Values are expressed as mean \pm standard deviation (SD) of three samples obtained from three independent experiments. Statistical comparisons were made using one-way analysis of variance (ANOVA) and multiple comparisons between groups were performed using Tukey's test. $P<0.05$ was considered statistically significant, while $P<0.01$ was highly significant.

\section{Results}

\subsection{Photosensitizers}

The structural formulae of the two Pcs are presented in Fig. 1. As we can see, both of the two Pcs have several phenoxy groups introduced at the macrocycle periphery, and the obtained phthalocyanines were then quaternized with different numbers of aminos (for ZnPc1) or ethanediamine groups (for ZnPc2). As a result quaternized $\mathrm{ZnPc} 2$ was more positively charged compared with ZnPc1, which may contribute a lot to its antimicrobial activity.

\subsection{Uptake of Pcs into the cell}

As shown in the Fig. 2, 2A and B demonstrated the fluorescence emission spectra after incubating Pcs with bacteria for different internals. The black curves represented the spectra of the mixture (bacteria and lysate) before adding Pcs. We could see that before adding drugs, the curves were flat. After adding Pcs, however, all the curves peaked while the wavelength was nearly $689 \mathrm{~nm}$. The relative fluorescence of the two Pcs both increased rapidly and reached peak after 40 minutes of incubation. After this time the fluorescence intensity declined gradually. Fig. 2C and $\mathrm{D}$ showed the maximum fluorescence quantization of Pcs over different time periods; both curves showed the same tendency during the processing. According to these results, 40 minutes was selected as the Pcs incubation time in the further experiments.

\subsection{Dark toxicity of Pcs to $E$. coli}

In order to identify whether Pcs itself has a killing effect on $E$. coli in the dark environment, we incubated the bacterial cells with various concentrations of Pcs. As demonstrated in Fig. 3, both Pcs had no obvious dark toxicity on E. coli at the Pcs concentrations that we chose in our experiments.

\subsection{Light toxicity studies}

Results are shown in Fig. 4. The highest light dose we used in this part was $200 \mathrm{~J} \mathrm{~cm}^{-2}$, which was four times the highest light dose we applied in photodynamic treatment; however, no evident decrease in the CFU counts was observed, indicating that the light irradiation itself would not kill the cells by heating (Fig. 4A). The membrane integrity detected by microscope is presented in Fig. 4B. From the pictures, we can see clearly that the number of cells with red fluorescence showed no obvious change even we increased the light dose to $200 \mathrm{~J} \mathrm{~cm}^{-2}$, which further proved that irradiation alone would cause no damage to bacteria. Furthermore, the temperature change monitored using a thermal imaging instrument showed that the illumination could not kill the bacteria by heating. Before illumination, the room temperature (0 minute) was about $35.8{ }^{\circ} \mathrm{C}$. 

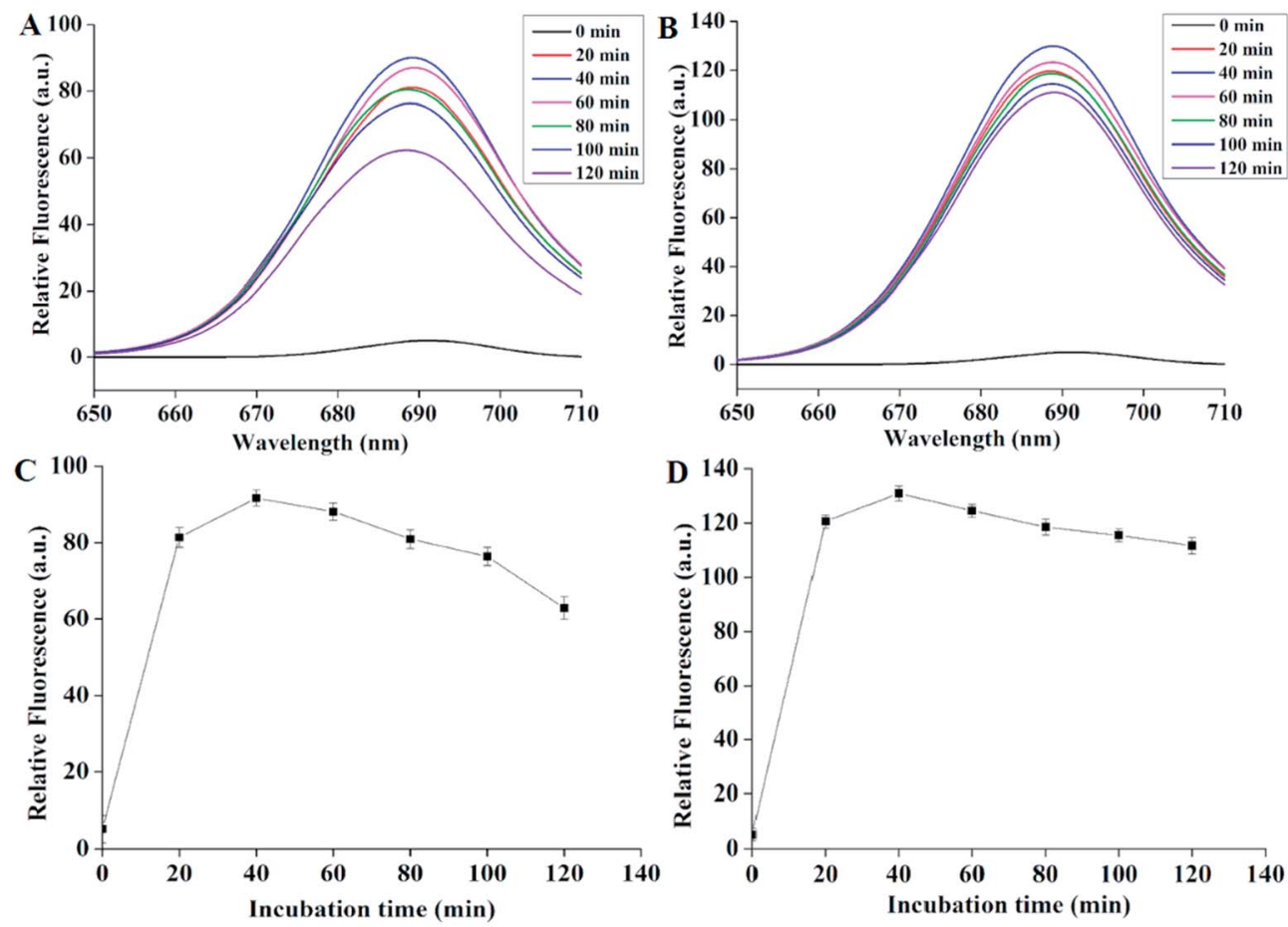

Fig. 2 Uptake of Pcs by Escherichia coli (E. coli). (A) Absorption spectrum of ZnPc1 ((B) for ZnPc2) after incubating with E. coli for different time periods. (C) Maximum fluorescence quantization of ZnPc1 ((D) for ZnPc2) over different time periods. Data are expressed as mean \pm SD of three independent experiments.
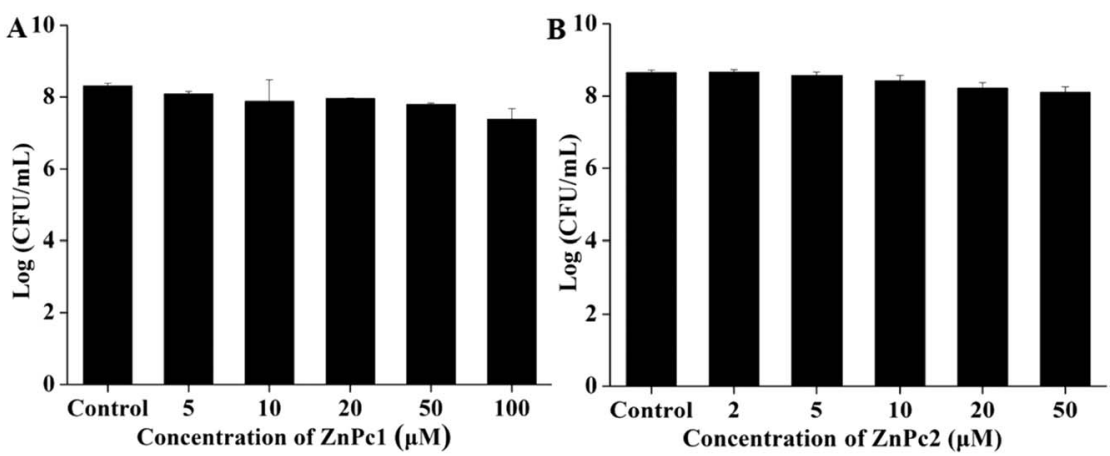

Fig. 3 Dark toxicity of PCs on E. coli. (A) The colony forming units (CFU) counts after incubating with different concentrations of ZnPc1 (0, 5, 10, 20, 50, and $100 \mu \mathrm{M})$. (B) The CFU counts after incubating with different concentrations of $\mathrm{ZnPc2}(0,2,5,10,20$, and $50 \mu \mathrm{M})$. Data are expressed as mean \pm SD of three independent experiments.

Although the temperature increased gradually over time during irradiation, it was lower than $38^{\circ} \mathrm{C}$ for the whole 10 minutes. In our Pcs-PACT studies, while the light dose was $50 \mathrm{~J} \mathrm{~cm}^{-2}$, the illumination time was less than 6 minutes. When the irradiation time was prolonged to 6 minutes, the temperature we measured was about $37.5{ }^{\circ} \mathrm{C}$ (Fig. 4C). Thus, we can conclude that the irradiation itself would not kill the cells during PcsPACT experiments, and the main factor to kill the cells is the photodynamic effect.

\subsection{Photodynamic antimicrobial activity of $\mathrm{ZnPc1}$ and $\mathrm{ZnPc2}$}

We chose three different concentrations of drug conjugates with three light doses to investigate the PACT sterilization intensity in detail in this part. To emphasize the antimicrobial effects in a light dose-dependent manner, we only showed the results of maximum concentrations combined with different light doses; the other data (while the concentration was 5, $10 \mu \mathrm{M}$ for $\mathrm{ZnPc} 1$ and 1, $2 \mu \mathrm{M}$ for $\mathrm{ZnPc} 2$ ) are not shown. It can be seen in Fig. 5 that the CFU counts of the two photosensitizers both decreased gradually with the increase of the light dose. For ZnPc1, the bactericidal effect appeared from $10 \mathrm{~J} \mathrm{~cm}^{-2}$ of light dose, and when the illumination dose increased to $50 \mathrm{~J} \mathrm{~cm}^{-2}$, a $3.10-\log$ reduction $(P<0.01)$ was obtained by photodynamic therapy with ZnPc1 at $20 \mu \mathrm{M}$ (Fig. 5A). For ZnPc2, both the drug concentrations and light doses applied were smaller compared with ZnPc1; however, while the light dose was only $20 \mathrm{~J} \mathrm{~cm}^{-2}$ 

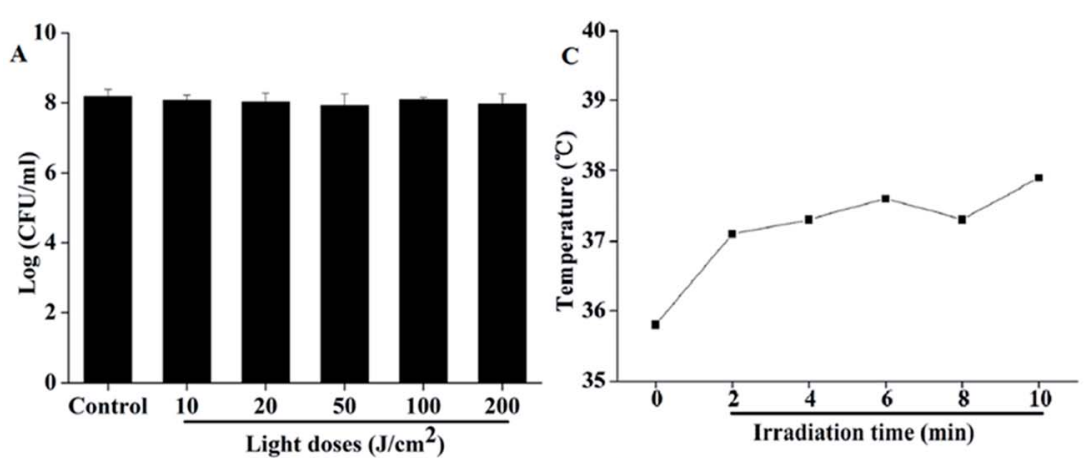

B

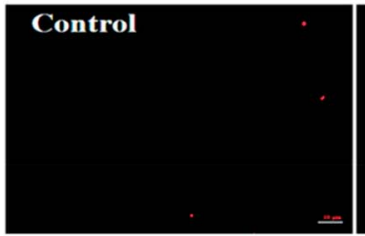

Light-10 J/cm²
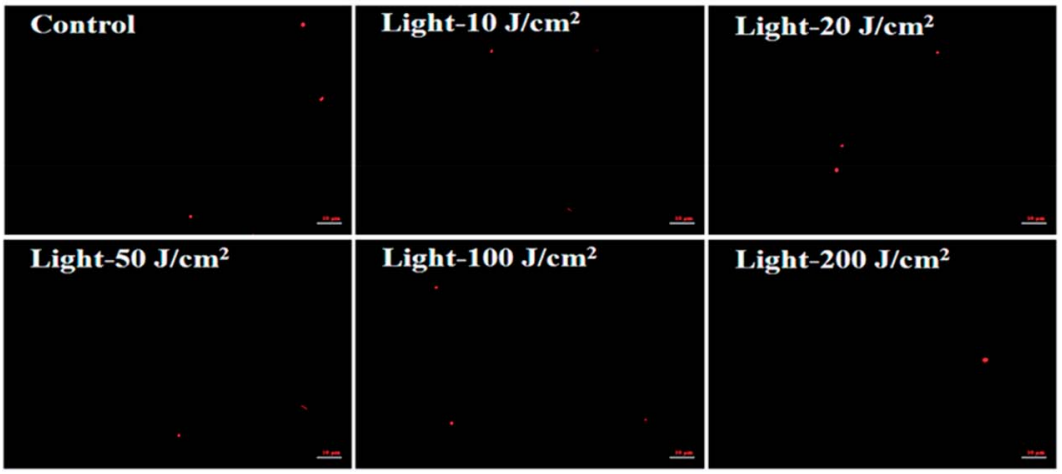

Fig. 4 Light toxicity on E. coli and temperature changing during irradiation. (A) The CFU counts after irradiating with different light doses (0, 10, $20,50,100,200 \mathrm{~J} \mathrm{~cm}^{-2}$ ). (B) Images taken by fluorescence microscope of $E$. coli treated with different doses of light. (C) Temperature change from 0 to 10 minutes detected by infrared thermal imaging instrument during irradiation. Data are expressed as mean \pm SD of three independent experiments.
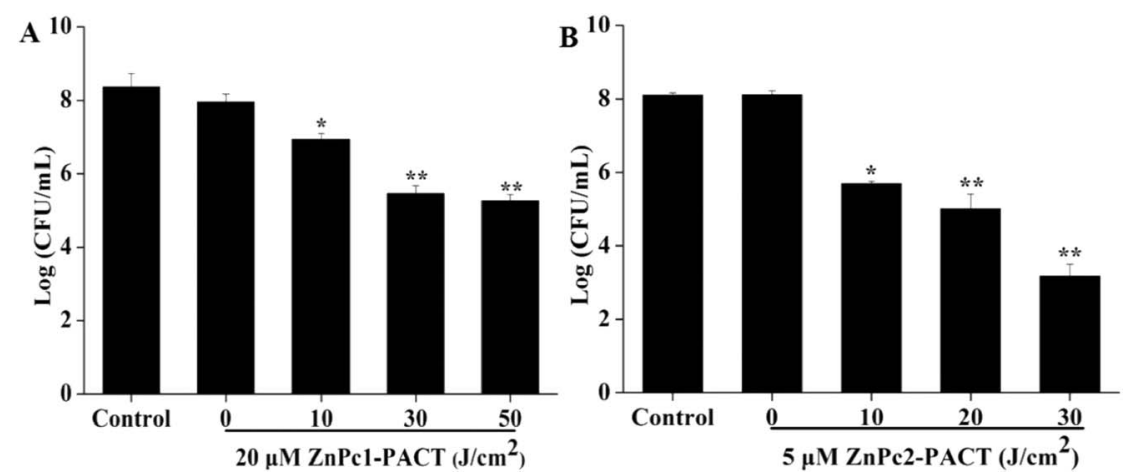

Fig. 5 Colony forming units assay of E. coli after PCS-PACT treatments. (A) The CFU counts after PACT treatment of $20 \mu M$ ZnPC1 with different light doses $\left(0,10,30\right.$, and $\left.50 \mathrm{~J} \mathrm{~cm}^{-2}\right)$. (B) The counts of CFU after PACT treatment of $5 \mu \mathrm{M} \mathrm{ZnPc2} \mathrm{with} \mathrm{different} \mathrm{light} \mathrm{doses}(0,10,20$, and $30 \mathrm{~J} \mathrm{~cm}^{-2}$ ). Data are expressed as mean \pm SD of three independent experiments. $* P<0.05, * * P<0.01$ versus control.

combined with Pcs at $5 \mu \mathrm{M}$, a nearly $3-\log$ reduction $(P<0.01)$ was attained, which was accomplished when the light dose was $50 \mathrm{~J} \mathrm{~cm}^{-2}$ for ZnPc1. When the illumination dose increased to $30 \mathrm{~J} \mathrm{~cm}^{-2}$, the $\log$ reduction was $4.91(P<0.01)$ (Fig. 5B). The results suggested that both Pcs had excellent antimicrobial activity in a light dose-dependent manner.

\subsection{ROS production}

The production of ROS was detected by flow cytometry. Because of the fluorescence quenching caused by excess luminance, we chose smaller light doses in this module $\left(1,2,5 \mathrm{~J} \mathrm{~cm}^{-2}\right.$ for $\mathrm{ZnPc1}$ and 2, 5, $10 \mathrm{~J} \mathrm{~cm}^{-2}$ for ZnPc2). It could be seen that in the control group and the ZnPc1 alone group only $1.49 \%$ and $1.39 \%$ cells showed red fluorescence, respectively. However, in the PACT groups, the bacteria showed markedly increased fluorescence: $41.50 \%(P<0.01)$ of cells displayed high DHE fluorescence when the light dose was $5 \mathrm{~J} \mathrm{~cm}^{-2}$ (Fig. 6A and B). For $\mathrm{ZnPc} 2$, we can see the same phenomenon with ZnPc1, and the fluorescence intensity increased to $57.59 \%(P<0.01)$ when the light dose was only $2 \mathrm{~J} \mathrm{~cm}^{-2}$. When the light dose of $10 \mathrm{~J} \mathrm{~cm}^{-2}$ was applied, $69.87 \%(P<0.01)$ of cells showed red fluorescence (Fig. 6C and D). The results echoed our previous studied very well and further proved that $\mathrm{ZnPc} 2$ had better antimicrobial activity than ZnPc1. 

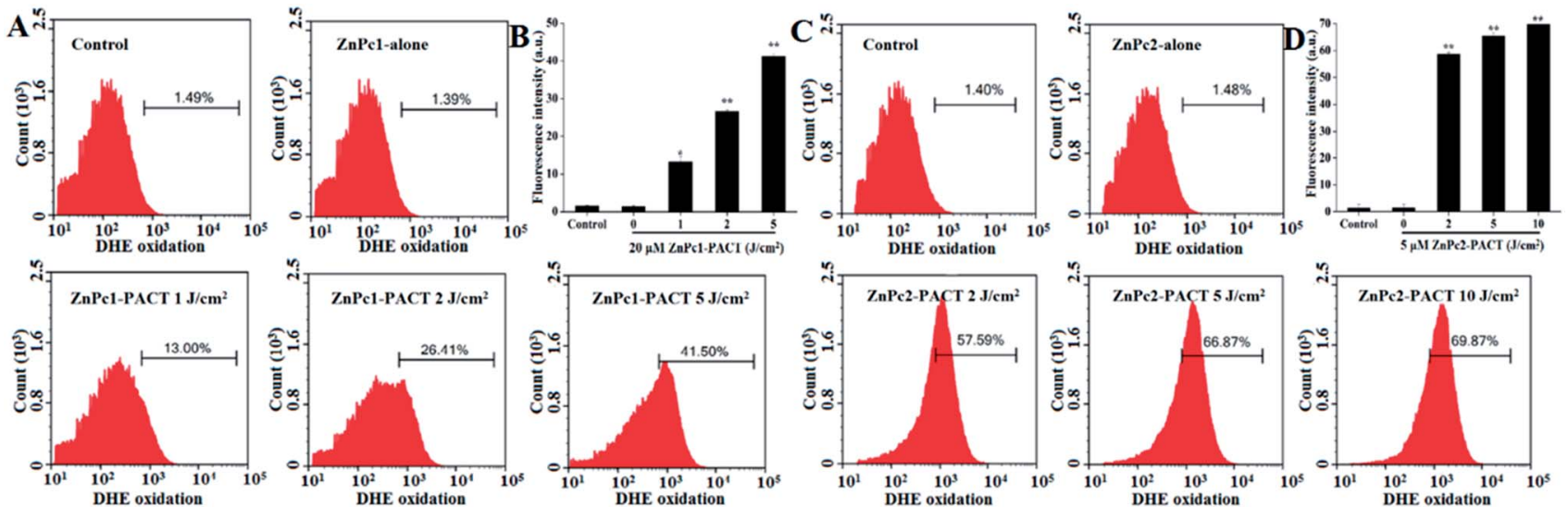

Fig. 6 ROS detection by flow cytometry measurement. (A) Cytofluorometric profiles representing the distribution of bacterial cells treated with $20 \mu \mathrm{M}$ ZnPc1-PACT ((C) is for $5 \mu \mathrm{M} \mathrm{ZnPc2)}$ after staining with DHE. (B) Fluorescence quantization in different groups treated with ZnPc1 ((D) is for $\mathrm{ZnPc2}$ ). Data are expressed as mean $\pm \mathrm{SD}$ of three independent experiments. $* P<0.05, * * P<0.01$ versus control.

\subsection{Membrane integrity}

Membrane integrity results are shown in Fig. 7. In the control groups, very few cells showed fluorescence (Fig. 7A and C), indicating that most cells had intact membrane structures. In the light alone and Pcs alone groups, the proportion of fluorescence cells showed no evident change compared with the control groups. However, the fluorescence intensity had a sharp increase in the PACT treatment groups. For ZnPc1, when the light dose was $50 \mathrm{~J} \mathrm{~cm}^{-2}$, nearly $42.00 \%$ cells showed red fluorescence, demonstrating that the photodynamic action of Pcs induced significant damage to the membrane integrity of $E$. coli (Fig. 7A and B). While the light dose continued to increase, the trend of increasing was not so evident; microscope images proved this phenomenon (Fig. 7E). For ZnPc2, the fluorescence
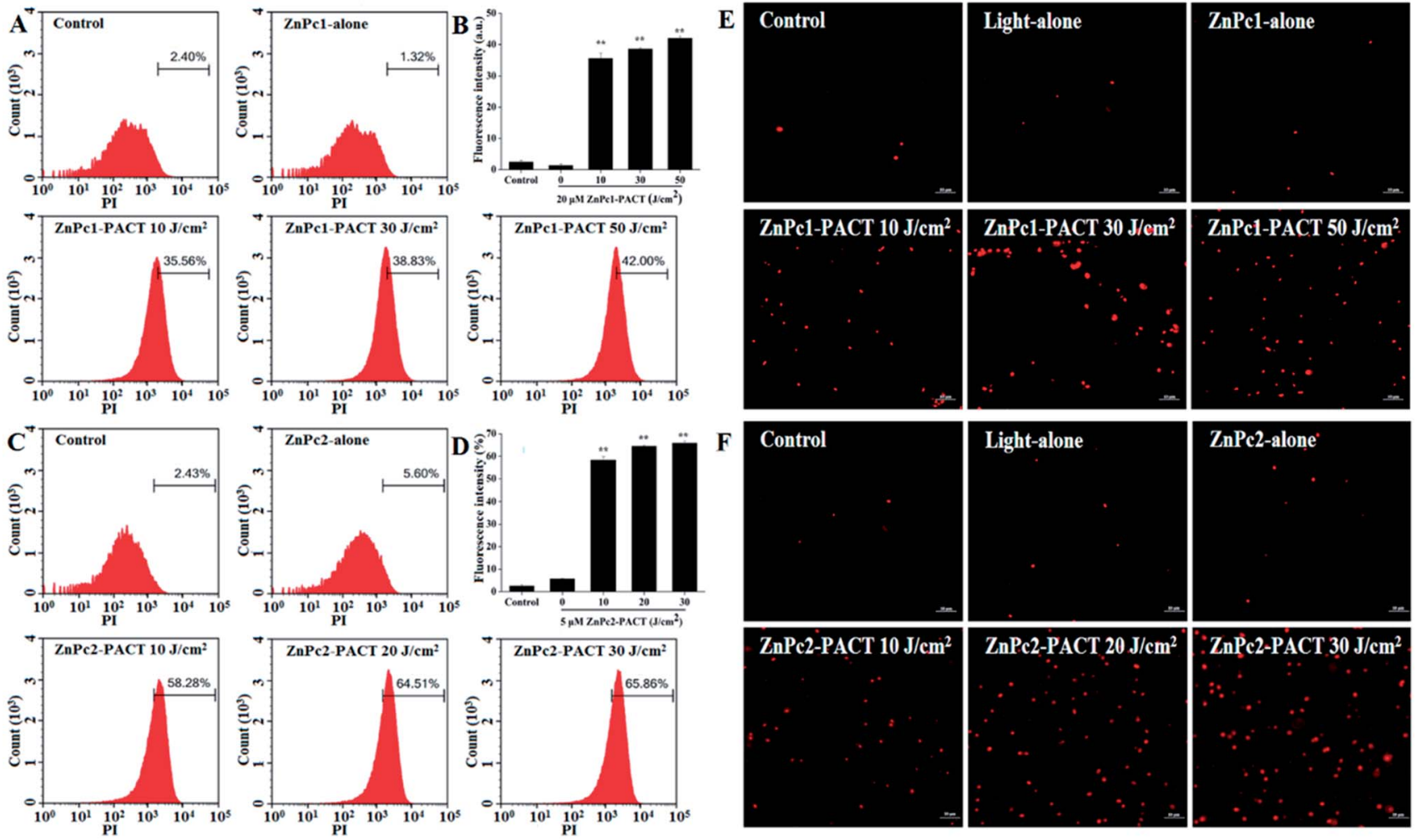

Fig. 7 Membrane integrity detected by PI staining. (A) Red fluorescence after PACT treatment with $20 \mu \mathrm{M} Z \mathrm{nPc1}$ combined with different doses of $0,10,30$, and $50 \mathrm{~J} \mathrm{~cm}^{-2}$. (B) Fluorescence quantization of (A). (C) Red fluorescence after PACT treatment with $5 \mu \mathrm{M}$ ZnPc2 combined with different doses of $0,10,20$, and $30 \mathrm{~J} \mathrm{~cm}^{-2}$. (D) Fluorescence quantization of (C). (E) Images taken by fluorescence microscope of $E$. coli treated with $20 \mu \mathrm{M}$ ZnPc1 in different groups. (F) Images taken by fluorescence microscope of $E$. coli treated with $5 \mu \mathrm{M} Z \mathrm{nPc} 2$ in different groups. Data are expressed as the mean \pm SD of three independent experiments. ${ }^{*} P<0.05,{ }^{*} P<0.01$ versus control. 

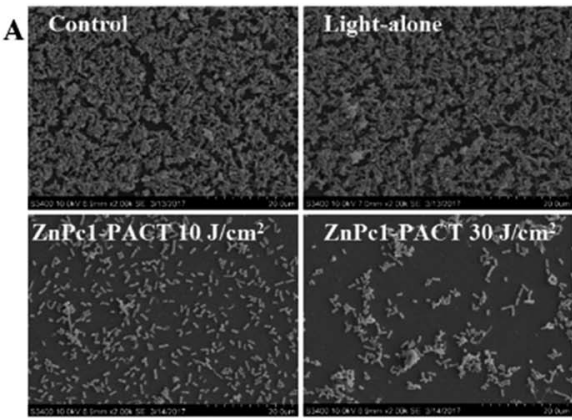
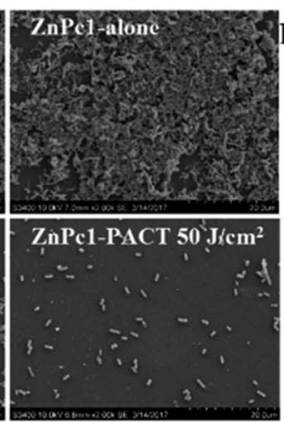

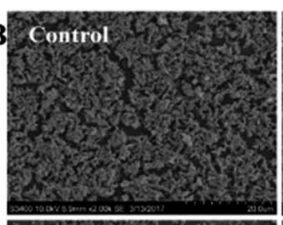

ZnPc2-PACT $10 \mathrm{~J} / \mathrm{em}$

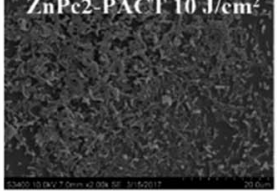

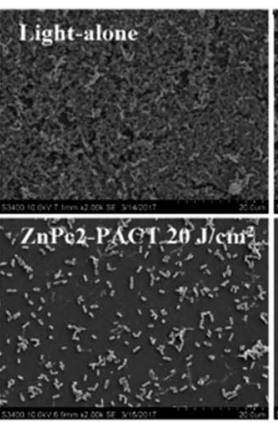

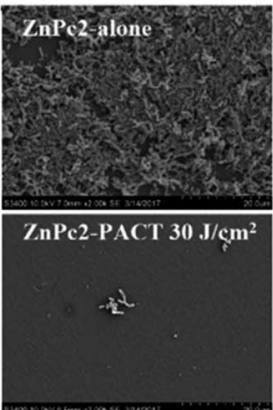

Fig. 8 SEM images of PACT-subjected E. coli biofilms. (A) Images of $E$. coli treated with $20 \mu M$ ZnPc1-PACT ((B) is for $5 \mu M$ ZnPc2) in different groups. Control: without any treatment; light alone: bacteria were treated with light alone; Pcs alone: bacteria were treated with $20 \mu \mathrm{M} Z \mathrm{nPc1}$ or $5 \mu \mathrm{M}$ ZnPc2 alone; ZnPc1-PACT $10 \mathrm{~J} \mathrm{~cm}^{-2}$ : ZnPc1 combined with $10 \mathrm{~J} \mathrm{~cm}^{-2}$ light; ZnPc1-PACT $30 \mathrm{~J} \mathrm{~cm}^{-2}: \mathrm{ZnPc1}_{\mathrm{combined} \mathrm{with} 30 \mathrm{~J} \mathrm{~cm}} \mathrm{co}^{-2}$ light; ZnPc1-PACT $50 \mathrm{~J} \mathrm{~cm}^{-2}$ : ZnPc1 combined with $50 \mathrm{~J} \mathrm{~cm}^{-2}$ light; ZnPc2-PACT $10 \mathrm{~J} \mathrm{~cm}^{-2}$ : ZnPc2 combined with $10 \mathrm{~J} \mathrm{~cm}^{-2}$ light; ZnPc2PACT $20 \mathrm{~J} \mathrm{~cm}^{-2}$ : ZnPc1 combined with $20 \mathrm{~J} \mathrm{~cm}^{-2}$ light; ZnPc1-PACT $30 \mathrm{~J} \mathrm{~cm}^{-2}$ : ZnPc2 combined with $30 \mathrm{~J} \mathrm{~cm}^{-2}$ light.

intensity peaked when the light dose was $30 \mathrm{~J} \mathrm{~cm}^{-2}$ (Fig. 7C and D). Images taken by fluorescence microscope further proved that the membrane was seriously damaged by photodynamic treatments (Fig. 7F).

\subsection{Photodynamic inactivation of $E$. coli biofilms}

To learn the effect of PACT on bacterial biofilm morphology, SEM studies were carried out. As we can see, bacteria in the control groups, light alone and Pcs alone groups, a large number of colonies formed a compact structure and showed no destruction in appearance. The biological membrane structure gradually relaxed and some bacteria even presented single colony distribution after different PACT treatments. At a dose of $50 \mathrm{~J} \mathrm{~cm}^{-2}$ in the presence of $20 \mu \mathrm{M} \mathrm{ZnPc1,} \mathrm{few} \mathrm{aggregated}$ colonies were observed in E. coli biofilms (Fig. 8A); the same effect was achieved in another Pcs ZnPc2 with a concentration of $5 \mu \mathrm{M}$ combined with a light dose of $20 \mathrm{~J} \mathrm{~cm}^{-2}$ (Fig. 8B). When the light dose increased to $30 \mathrm{~J} \mathrm{~cm}^{-2}$, there were nearly no colonies formed, suggesting an absence of classic biofilm morphology. These results indicated that Pcs-PACT can affect the structure of the biofilm and adhesion between the bacteria.

\section{Discussion}

Microbial infections remain one of the main causes of mortality worldwide. Due to the abuse of antibiotics, microbes have adopted a large variety of mechanisms to increase their resistance towards antibiotics. ${ }^{36,37}$ Such conditions necessitate new methods to be developed to treat microorganism infections, especially those caused by drug-resistant strains. Photodynamic antimicrobial chemotherapy (PACT) is a new method to do this, which combines photodynamic irradiation with drugs known as sensitizers to treat both cancers and infections of pathogenic microorganisms, and has produced good results. ${ }^{13-16}$

In this study, we chose two cationic phthalocyanines (Pcs), $\mathrm{ZnPc1}$ and $\mathrm{ZnPc} 2$, as photosensitizers to combine with light to measure their antibacterial activity. Compared with other photosensitizers, our Pcs presented better antimicrobial activity. Managa et al. reported that using (5,10,15,20-tetrakis(4- (4-carboxyphenycarbonoimidoyl)phenyl)porphyrinato)chloro gallium(III)-conjugated platinum nanoparticles combined with PACT caused only a 3.76-log reduction to Escherichia coli (E. coli) counts. ${ }^{16}$ In addition, Perni et al. demonstrated that silica-TBO nanoconjugates combined with light showed a slight antimicrobial effect against E. coli. ${ }^{38}$ In our study, however, Pcs showed excellent sterilization efficiency in both planktonic and biofilm states.

To investigate whether the modified Pcs could be absorbed effectively by bacterial cells, we first tested the uptake of the drug. According to our pretest study, at low concentrations, fluorescence could not be detected, and the difference in uptake between the two drugs could not be revealed. In order to measure the uptake under the same concentration, we chose the concentration of $5 \mu \mathrm{M}$ for both Pcs to be detected. The results indicated that the two Pcs had the same tendency. In the first 40 minutes, the drug was rapidly absorbed by the cells, and the fluorescence intensity increased quickly and reached a peak at 40 minutes. After this period, the absorption curves showed a downward trend, probably because of the drug extravasation (Fig. 2). In view of such condition, 40 minutes was selected as the best incubation time in later experiments. Besides, we could see that the absorption peak of $\mathrm{ZnPc} 2$ was larger than that of ZnPc1, and our previous studies demonstrated that the intracellular uptake efficacy of Pcs is directly related to the number of positive ions in their structures. ${ }^{23} \mathrm{ZnPc} 2$ was more positively charged, which made it easier to bind with the negative-charged bacteria membrane, as the charge number increased the amphiphilic character of the photosensitizers, promoting the drug binding with the cell membrane and accumulating in the cells. ${ }^{39-41}$ As a result, the cells absorbed more $\mathrm{ZnPc} 2$ than $\mathrm{ZnPc1}$ at the same concentration. According to the results, the doses of ZnPc2 used in the following experiments were smaller compared with ZnPc1.

Considering that the dark environment and light irradiation applied in the experiments may cause damage to the bacteria, the plate count method was employed to detect the dark and light toxicity of the two Pcs. To detect the dark toxicity, we first chose the same concentration for two Pcs ranging from 0 to 
$100 \mu \mathrm{M}$. When the concentration was $100 \mu \mathrm{M}$, there was no obvious dark toxicity of ZnPc1 while ZnPc2 showed mild dark toxicity to E. coli cells, so we lowered the concentrations for ZnPc2. The results showed no obvious damage occurred at the chosen concentration range (Fig. 3). Furthermore, illumination itself had no bactericidal effect because no evident decrease in the CFU counts was shown and the membrane integrity showed no change even when the light dose was increased to $200 \mathrm{~J}$ $\mathrm{cm}^{-2}$. Besides, the temperature change results during irradiation showed that the temperature was less than $38{ }^{\circ} \mathrm{C}$ over 10 minutes (Fig. 4). Fotadar et al. reported that $E$. coli can grow consistently at a temperature as high as $49{ }^{\circ} \mathrm{C},{ }^{42}$ so it could be concluded that the heating caused by irradiation alone would not kill the bacteria in this study. After photodynamic treatment, however, the antimicrobial effect increased sharply. In our pretest experiments, we found that the antimicrobial activity was both light dose- and concentration-dependent. For $\mathrm{ZnPc2}$, when the light dose increased to $50 \mathrm{~J} \mathrm{~cm}^{-2}$, there were no viable cells when the concentration was $5 \mu \mathrm{M}$, so we selected smaller light doses compared with ZnPc1. Nearly 3.1- and 5-log reductions in bacterial numbers were achieved while the peak concentrations and maximum doses were applied for ZnPc1 and $\mathrm{ZnPc2}$, respectively (Fig. 5). The results revealed that the modified cationic Pcs had excellent antimicrobial activity after combining with light. Whether smaller concentrations of Pcs combined with higher light doses could cause obvious damage to bacteria is worth investigating in future study.

It is well known that oxidation stress is one of the key factors in the cell killing of PACT. ${ }^{43}$ In order to determine whether it was the intercellular reactive oxygen species (ROS) that played an important role, the intercellular ROS level was measured using a superoxide anions fluorescence probe dihydroethidium (DHE). According to the results of photodynamic antimicrobial experiments, we chose two concentrations at which the best antimicrobial effect was shown ( $20 \mu \mathrm{M}$ for $\mathrm{ZnPc} 1$ and $5 \mu \mathrm{M}$ for $\mathrm{ZnPc} 2)$ to compare the ROS production. When choosing light doses, we first used the same light doses applied in former parts $(10,30$, and $50 \mathrm{~J} \mathrm{~cm}^{-2}$ for $\mathrm{ZnPc1}$ and 10, 20, and $30 \mathrm{~J} \mathrm{~cm}^{-2}$ for $\mathrm{ZnPc} 2$ ) in our pretest study. However, because of the fluorescence quenching caused by excess luminance, the fluorescence detected by flow cytometry was very low, so the light doses were changed for both Pcs $\left(1,2,5,10,30\right.$, and $50 \mathrm{~J} \mathrm{~cm}^{-2}$ for ZnPc1 and $1,2,5,10,20$, and $30 \mathrm{~J} \mathrm{~cm}^{-2}$ ). For ZnPc1, the fluorescence intensity increased with increasing light dose and peaked at a light dose of $5 \mathrm{~J} \mathrm{~cm}^{-2}$; as the light dose continued to increase, the fluorescence intensity declined. For $\mathrm{ZnPc} 2$, the same situation was observed and the fluorescence peaked at a light dose of $10 \mathrm{~J} \mathrm{~cm}^{-2}$. In our results, we only intercepted the part in which the fluorescence intensity increased with increasing light dose (Fig. 6). Previous research has shown that charge can influence singlet oxygen generation ability, thereby affecting their photodynamic activity, ${ }^{24}$ which can also explain why ZnPc2 had better antimicrobial activity compared with ZnPc1 in our study. The ROS-mediated bactericidal mechanism has been verified in a lot of studies. Carrasco et al. and Valko et al. demonstrated that ROS can cause damage to polyunsaturated proteins, ${ }^{33}$ amino acids and lipids, ${ }^{44}$ which means that membranes composed mainly of lipids are possible targets of PACT.

In order to test the effect of Pcs-PACT on membrane integrity, propidium iodide (PI) staining was employed. Very few cells showed red fluorescence in the control, light alone and Pcs alone groups, but the proportion of fluorescence cells had a sharp increase in a light dose-dependent manner in the PACT treatment groups, suggesting that Pcs-PACT could induce serious damage to membrane integrity (Fig. 7A-D). Another phenomenon observed both in microscope images and flow cytometry was that as the light dose increased, the trend of fluorescence increase was not so evident (Fig. 7E and F). The reason may be that the membrane was only one of the targets of PACT but not the sole target, so we could not see an obvious change of fluorescence in this part, but what we assume was that $\mathrm{ZnPc2}$ had better antimicrobial activity compared with $\mathrm{ZnPc1}$.

Biofilm is another factor that affects the effect of PACT; it is aggregates of microbial cells composed mainly of a largemolecular-weight polysaccharides and embedded in a selfproduced matrix of extracellular polymeric substances (EPS), ${ }^{35}$ which is an important component of biofilms and can provide a protective environment to isolate the cells from adverse environmental conditions, such as acid, ultraviolet radiation and antibiotics, ${ }^{35,45}$ thus making it more difficult to kill bacteria in biofilm-producing mode. Many researchers have made great efforts to develop new drugs with special properties to overcome the biofilm resistance of bacteria. Liu et al. reported a kind of nanomaterial that could bypass biofilm recalcitrance to antimicrobial penetration but was subjected to $\mathrm{pH}$ change, ${ }^{35}$ which may weaken its impact in neutral or alkaline conditions. Liu et al. once reported in a paper that combining hematoporphyrin monomethyl ether (HMEM) at a concentration at $100 \mu \mathrm{M}$ with light irradiation could only cause a 4.01-log reduction in the survival of $E$. coli in the biofilm state. ${ }^{13}$ In view of the two chemically modified cationic Pcs applied in this study had an excellent sterilization effect, the Pcs-PACT effect of $E$. coli in the biofilm form was also evaluated. To compare the anti-biofilm effects at the same level, the concentrations and light doses applied were in line with the experimental conditions we used in former parts. Compared with the control and Pcs alone groups in which cells showed no destruction in appearance and agglomerate states, the cells in the Pcs-PACT groups presented single colony distribution. In the presence of $20 \mu \mathrm{M} \mathrm{ZnPc1}$ combined with $50 \mathrm{~J} \mathrm{~cm}^{-2}$ light illumination or $5 \mu \mathrm{M} \mathrm{ZnPc2}$ combined with a light dose of $30 \mathrm{~J} \mathrm{~cm}^{-2}$, nearly no aggregated colonies were observed in E. coli biofilms, indicating that both ZnPc1 and ZnPc2 had excellent antimicrobial effects in the biofilm state (Fig. 8).

On the whole, our experiments have compared the antimicrobial activity from several different levels, and all of the results showed that $\mathrm{ZnPc} 2$ had better antimicrobial activity than ZnPc1. Here, we can give a brief explanation to declare the photodynamic activity difference between the two Pcs according to our results. Since $\mathrm{ZnPc} 2$ had both higher cellular uptake and ROS generation efficiency, it had better antibacterial activity compared to ZnPc1. An important factor that contributed to the higher cellular uptake of $\mathrm{ZnPc} 2$ was its more positive charge, 
suggesting that the cationic property is important for photosensitizers to exert their effect. Liu et al. reported a kind of surface charge-switching nanoparticles with improved treatment efficacy and reduced probability of drug resistance because it increased the cell wall targeting of the photosensitizers. ${ }^{41}$ Alves et al. demonstrated that the photodynamic efficiency of cationic porphyrins in the photosensitization of E. coli and Enterococcus faecalis increased with the number of charges, ${ }^{\mathbf{4 6}}$ because the charge number could increase the amphiphilic character of the photosensitizers, and its affinity for bacteria increased as a result, which would help the drug to accumulate in the cells..$^{37,39,41}$ Moreover, quaternized ions significantly increased the ability of $\mathrm{ZnPc} 2$ to produce singlet oxygen, which played an important role in killing cells. ${ }^{23}$ All of the elements worked together and finally enhanced the photodynamic activity.

Apart from what we have done in our experiments, other papers have reported that ROS can irreversibly modify DNA molecules, severely disturb DNA replication and transcription mechanisms, and finally kill the cells. ${ }^{47-49}$ Thus means that DNA may be an important target of PACT. However, in this study we did not do the related research. Further research is needed to affirm this point and elucidate the antimicrobial mechanisms of the two Pcs and investigate other chemical modifications of the most promising Pcs in order to obtain molecules that are potentially suitable for in vivo and clinical application.

\section{Conclusions}

The present experiments show that both the Pcs used in this study have a significant inactivation effect on $E$. coli in combination with PACT, and ZnPc2 exhibited better antimicrobial activity compared with ZnPc1. Further in vivo experiments and mechanism exploration are needed to confirm that Pcs can meet all the requirements for the use of PACT as an alternative option to antibiotic treatments in clinical practice.

\section{Conflicts of interest}

The authors have declared that no conflict of interest exists.

\section{Acknowledgements}

This research was supported by the National Natural Science Foundation of China (No. 81472846, No. 81571834 and No. 21671105), the Natural Science Foundation of Shaanxi Province (No. 2017JM8004), Fundamental Research Funds for the Central Universities (No. GK201602003, No. GK201701005 and No. 2017CBY006), Shaanxi Normal University Training Programs of Innovation and Entrepreneurship for Undergraduates (No. cx17117), and National College Students' Innovative Program (KY2017ZD020).

\section{References}

1 E. Darabpour, N. Kashef and S. Mashayekhan, Photodiagn. Photodyn. Ther., 2016, 14, 211-217.
2 U. Römling and C. Balsalobre, J. Intern. Med., 2012, 272, 541561.

3 F. Sun, F. Qu, Y. Ling, P. Mao, P. Xia, H. Chen and D. Zhou, Future Microbiol., 2013, 8, 877-886.

4 W. W. Wu, M. M. Benjamin and G. V. Korshin, Water Res., 2001, 35, 3545-3550.

5 W. Yang, H. Zhou and N. Cicek, Crit. Rev. Environ. Sci. Technol., 2014, 44, 1443-1476.

6 X. Wang, M. Ip, A. W. Leung and C. Xu, Ultrasonics, 2014, 54, 2109-2114.

7 J. Cama, H. Bajaj, S. Pagliara, T. Maier, Y. Braun, M. Winterhalter and U. F. Keyser, J. Am. Chem. Soc., 2015, 137, 13836-13843.

8 D. T. Vo and C. K. Lee, Carbohydr. Polym., 2017, 164, 109-117.

9 M. R. Hamblin and G. Jori, Photodynamic Inactivation of Microbial Pathogens: Medical and Environmental Applications, 2011.

10 T. Maisch, R. M. Szeimies, G. Jori and C. Abels, Photochem. Photobiol. Sci., 2004, 3, 907-917.

11 C. Spagnul, L. C. Turner and R. W. Boyle, J. Photochem. Photobiol., B, 2015, 150, 11-30.

12 H. C. Flemming and J. Wingender, Nat. Rev. Microbiol., 2010, 8, 623-633.

13 C. Liu, M. Hu, D. Ma, J. Lei and J. Xu, Laser Med. Sci., 2016, 31, 297-304.

14 L. Zhai and K. W. Yang, Dyes Pigm., 2015, 120, 228-238.

15 Z. Chen, S. Zhou, J. Chen, L. Li, P. Hu, S. Chen and M. Huang, J. Lumin., 2014, 152, 103-107.

16 M. Managa, E. K. Amuhaya and T. Nyokong, Spectrochim. Acta, Part A, 2015, 151, 867-874.

17 D. M. Vera, M. H. Haynes, A. R. Ball, T. Dai, C. Astrakas, M. J. Kelso, M. R. Hamblin and G. P. Tegos, Photochem. Photobiol., 2012, 88, 499-511.

18 C. M. Cassidy, R. F. Donnelly and M. M. Tunney, J. Photochem. Photobiol., B, 2010, 99, 62-66.

19 I. Ü. M. Beyreis, N. Tortik, S. Z. Topal, M. Glueck, V. Ahsen, F. Dumoulin, T. Kiesslich and K. Plaetzer, Photodiagn. Photodyn. Ther., 2016, 13, 40-47.

20 D. Bechet, P. Couleaud, C. Frochot, M. L. Viriot, F. Guillemin and M. Barberi-Heyob, Trends Biotechnol., 2008, 26, 612-621.

21 J. P. Keene, D. Kessel, E. J. Land, R. W. Redmond and T. G. Truscott, Photochem. Photobiol., 1986, 43, 117-120.

22 D. Lazzeri, M. Rovera, L. Pascual and E. N. Durantini, Photochem. Photobiol., 2004, 80, 286-293.

23 A. Wang, L. Zhou, K. Fang, L. Zhou, Y. Lin, J. Zhou and S. Wei, Eur. J. Med. Chem., 2012, 58C, 12-21.

24 A. Wang, Y. Li, L. Zhou, L. Yuan, S. Lu, Y. Lin, J. Zhou and S. Wei, J. Photochem. Photobiol., B, 2014, 141, 10-19.

25 J. W. Hofman, Z. F. Van, S. Turker, H. Talsma, S. A. Lambrechts, D. V. Sakharov, W. E. Hennink and C. F. van Nostrum, J. Med. Chem., 2007, 50, 1485-1494.

26 F. Dumoulin, M. Durmuss, V. Ahsen and T. Nyokong, Coord. Chem. Rev., 2010, 254, 2792-2847.

27 D. Lin, Y. Wang, Q. Zhang, J. Zhou, L. Zhou and S. Wei, J. Photochem. Photobiol., A, 2016, 315, 107-120.

28 M. Durmuş, H. Yaman, C. Göl, V. Ahsen and T. Nyokong, Dyes Pigm., 2011, 91, 153-163. 
29 S. Haberl, M. Jarc, A. Strancar, M. Peterka, D. Hodžić and D. Miklavčič, J. Membr. Biol., 2013, 246, 861-867.

30 S. Choi, H. Lee, J. Yu and H. Chae, J. Photochem. Photobiol., B, 2015, 153, 7-12.

31 C. Brana, C. Benham and L. Sundstrom, Brain Res. Protoc., 2002, 10, 109-114.

32 D. J. Novo, N. G. Perlmutter, R. H. Hunt and H. M. Shapiro, Antimicrob. Agents Chemother., 2000, 44, 827-834.

33 E. Carrasco, A. Blázquezcastro, M. I. Calvo, Á. Juarranz and J. Espada, Methods, 2016, 109, 180-189.

34 H. Zhao, S. Kalivendi, H. Zhang, J. Joseph, K. Nithipatikom, J. Vásquezvivar and B. Kalyanaraman, Free Radical Biol. Med., 2003, 34, 1359-1368.

35 Y. Liu, H. J. Busscher, B. Zhao, Y. Li, Z. Zhang, V. D. M. Hc, Y. Ren and L. Shi, ACS Nano, 2016, 10, 4779-4789.

36 L. Misba, S. Zaidi and A. U. Khan, Photodiagn. Photodyn. Ther., 2017, 18, 24-33.

37 M. Vimaladevi, K. C. Divya and A. Girigoswami, J. Photochem. Photobiol., B, 2016, 162, 146-152.

38 S. Perni, S. Drexler, S. Ruppel and P. Prokopovich, Colloids Surf., A, 2016, 510, 293-299.

39 A. Minnock, D. I. Vernon, J. Schofield, J. Griffiths, J. H. Parish and S. B. Brown, Antimicrob. Agents Chemother., 2000, 44, 522-527.
40 C. Simões, G. Ncm, N. Mgpms, Â. Cunha, J. C. Tomé, A. C. Tomé, J. A. A. Cavaleiro and M. A. F. Faustino, Catal. Today, 2016, 266, 197-204.

41 S. Liu, S. Qiao, L. Li, G. Qi, Y. Lin, Z. Qiao, H. Wang and C. Shao, Nanotechnology, 2015, 26, 495602.

42 U. Fotadar, P. Zaveloff and L. Terracio, J. Basic Microbiol., 2005, 45, 403-404.

43 P. Wang, C. Li, X. Wang, W. Xiong, X. Feng, Q. Liu, A. W. Leung and C. Xu, Ultrason. Sonochem., 2015, 23, 116127.

44 M. Valko, D. Leibfritz, J. Moncol, M. T. Cronin, M. Mazur and J. Telser, Int. J. Biochem. Cell Biol., 2007, 39, 44-84.

45 X. Li, H. Guo, Q. Tian, G. Zheng, Y. Hu, Y. Fu and H. Tan, J. Surg. Res., 2013, 184, 1013-1021.

46 E. Alves, L. Costa, C. M. Carvalho, J. P. Tomé, M. A. Faustino, M. G. Neves, A. C. Tomé, J. A. Cavaleiro, Â. Cunha and A. Almeida, BMC Microbiol., 2009, 9, 70-82.

47 G. Bertoloni, F. M. Lauro, G. Cortella and M. Merchat, Biochim. Biophys. Acta, Gen. Subj., 2000, 1475, 169-174.

48 H. Abe and S. J. Wagner, Photochem. Photobiol., 1995, 61, 402-409.

49 M. B. Spesia, D. A. Caminos, P. Pons and E. N. Durantini, Photodiagn. Photodyn. Ther., 2009, 6, 52-61. 